\title{
REM: A Fast Slewing Robotized Telescope to Catch Near-InfraRed Afterglow of GRBs
}

\section{A. Antonelli}

INAF - Osservatorio Astronomico di Roma, Via Frascati, 33, 00040

Monte Porzio Catone, Italy. e-mail: angelo@mporzio.astro.it

F. M. Zerbi, G. Chincarini, G. Ghisellini

INAF - Osservatorio Astronomico di Brera, via Bianchi 46, 23807, Merate, Italy

M. Rodonò, G. Cutispoto

INAF - Osservatorio Astrofisico di Catania, Via S.Sofia 78, 95123 Catania, Italy

P. Conconi, S. Covino, E. Molinari

INAF - Osservatorio Astronomico di Brera, via Bianchi 46, 23807, Merate, Italy

G. Tosti

Universitá di Perugia, Piazza Università 1, 06100 Perugia, Italy

L. Nicastro

IASF - CNR, Via Ugo La Malfa 153, 90146 Palermo, Italy

E. Palazzi

IASF - CNR, via P. Gobetti 101, 40129 Bologna, Italy

on behalf of the REM Team.

\begin{abstract}
.
REM (Rapid Eye Mount) is a fully robotized fast slewing telescope equipped with a high throughput Near InfraRed (Z', J, H, K') camera (REMIR) and an optical slitless spectrograph (ROSS). A dedicated software for data reduction and software (AQuA) has been developed to extract scientific information from REM images without any human intervent. REM is installed in La Silla (Chile) and dedicated to detect and study the prompt optical/IR afterglow of Gamma Ray Bursts with the ambitious project of discovering objects at extremely high redshift. The synergy between REMIR camera and ROSS makes REM a powerful observing tool for any kind of fast transient phenomena.
\end{abstract}




\section{Introduction}

The discovery of the afterglow of Gamma Ray Bursts (GRBs) (Costa et al., 1987; Van Paradijs et al., 1987) allowed to fix the problem of their distances, but fundamental questions about the origin and the physics of GRBs are still open. Observations of the prompt afterglow of GRB events are of paramount importance for both GRB science and cosmology. Following this idea many space borne observatories are entirely (HETE II, Swift) or partly (AGILE, INTEGRAL) dedicated to detect GRBs and to send promptly a trigger with the position of the event. So a fast response ground-based follow-up facility should be in this sense highly desirable. The aim of such a facility such as a robotic telescope, would be useful both to record relevant information on the early afterglow and to activate Target of Opportunity procedure at larger telescopes.

\section{REM and Gamma Ray Bursts}

Gamma Ray Bursts are bright, transient events in the $\gamma$-ray sky, unpredictable in time and location, with a typical duration of the order of seconds. The burst event is immediately followed by a fading emission observable at all wavelengths called afterglow. If $95 \%$ of GRBs show a X-ray afterglow almost $50 \%$ of them do not show any optical afterglow at all. The lack of optical afterglow can be addressed to very different phenomena. In fact, the simultaneous detection of an afterglow in the IR band and the non-detection in the optical could directly flag the presence of a high-z object. If a burst is at high-z $(8<z<15)$, Ly$\alpha$ absorption dumps all the light at optical wavelengths. On the contrary, if the burst happens at low redshifts the lack of optical afterglow can be due to absorption by intervening matter (dust), either in the close vicinity of the burst (if exploded in a dense star forming region whose dust has not been completely destroyed by the burst emission itself), or by dust distributed along the line of sight, even at large distances from the burst site. In this case the infrared light is much less absorbed, and therefore an IR transient can be detected even if the optical is not providing important information on the circumburst matter.

In all that cases in which an optical afterglow has been detected the typically monochromatic flux of it decreases in time as a power law $F_{\nu}(t) \sim t^{-\delta}$ with $\delta$ in the range 0.8-2. Typical magnitudes of optical afterglow detected about one day after the $\gamma$-ray event are in the range 19-21. Assuming $\mathrm{m}=19$ after 24 hours and $\delta \sim 1.5$, the expected magnitude after 1 hour is $\sim 13.8$. Although we have only two examples of prompt optical/IR emission during the first minutes of the afterglow (GRB 990123 and GRB 020813) they substantially confirmed our estimations of typical early magnitudes of GRBs afterglow. GRB 990123 has been detected by the robotic telescope ROTSE 22 seconds after the $\gamma$-ray trigger at $m \sim 11.7$, reaching $m \sim 8.947$ seconds after the trigger (Akerlof \& McKay 1999). GRB 021004 has been detected by the robotic telescope 48-inch Oschin/NEAT 567 seconds after the $\gamma$-ray trigger at $R m a g=15.34$ (Fox, 2002, GCN \#1564).

REM telescope with its NIR camera is expected to reach magnitude $\mathrm{H}=15.5$, 16.04 and 17.11 with exposure times of 5,30 and 600 seconds respectively $(\mathrm{S} / \mathrm{N}=5)$. With the ROSS spectrograph a $\mathrm{V}=14$ point-like source is recorded 

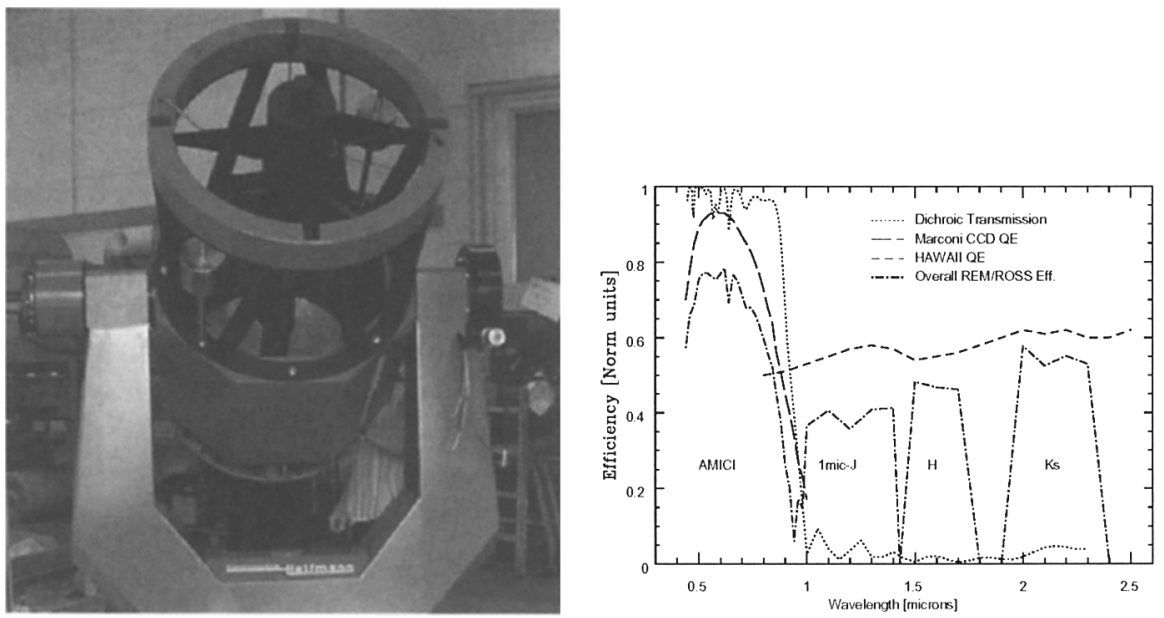

Figure 1. The REM Telescope and overall efficiency of the REM assembly: Dichroic, REMIR and ROSS.

better than $10 \sigma$ in $1 \mathrm{sec}$ exposures. The above numbers suggest that we can detect the IR afterglow during the first 2-4 hours even with an exposure time of 5 seconds. This will allow the study of the light curve and eventual flickering in great detail, the detection of possible (even if short) variations from the smooth power-law behavior and the definition of any possible break. Increasing the exposure time (after the initial phases) we can follow typical bursts up to 12 hours, after which larger telescopes can take over.

\section{REM Telescope and Instrumentation}

The REM telescope (Zerbi et al. 2002) is a Ritchey-Chretien system with a $60 \mathrm{~cm} \mathrm{f} / 2.2$ primary and a overall $\mathrm{f} / 8$ focal ratio mounted in an alt-azimuth mount in order to provide stable Nasmyth de-rotated focal stations, suitable for fast motions. At the first focal station (the second is idle) a dichroic, working at 45 degrees in the $\mathrm{f} / 8$ convergent beam, will split the beam to feed the two instruments of the REM telescope: the REM-IR camera and the ROSS Spectrograph. The telescope has been manufactured by Halfmann Teleskoptechnik $\mathrm{GmbH}$ (HT) (Augsburg, Germany). Mirrors are made by Zeiss and they are coated with protected silver to maximize reflection efficiency in such a large $(0.45-2.3 \mu)$ wavelength range.

The REM IR camera (e.g. Vitali et al., 2002) follows a focal reducer design in order to reform a white pupil in a cold $(77 \mathrm{~K})$ environment for Lyot-stop positioning. A filter wheel with 10 positions is located at the reformed pupil allowing one to insert filters and grisms for slit-less spectroscopy in a parallel beam. The camera changes the focal ratio from $\mathrm{f} / 8$ to $\mathrm{f} / 5.3$ providing a platescale of $64.4 \mathrm{as} / \mathrm{mm}$ that allows one to position a $9.9 \times 9.9 \mathrm{am}^{2}$ FOV on a $512 \times 512(18 \mu \mathrm{m}$ pitch) HAWAII HgCdTe chip in production at Rockwell. 
With an orthogonal development relative to the REMIR camera optical axis REM Nasmyth A will accommodate the slitless spectrograph ROSS (Zerbi et al., 2002). The spectrograph consists of a fore optics which images a pupil at the location of the dispersing element and re-maps the focal plane onto the detector unit. The selected detector head is a commercial Apogee AP47 camera hosting a Marconi $47-101 \mathrm{~K} \times 1 \mathrm{~K} 13 \mu \mathrm{m}$ pitch CCD. The plate scale of the REM telescope (43 $\mathrm{arcsec} / \mathrm{mm})$ matches properly with the specifications and allows one to cover a $9.54 \times 9.54 \mathrm{am}^{2}$ with a scale of $0.56 \mathrm{as} / \mathrm{px}$. The fore-optics have been then designed at magnification 1 . The dispersion is obtained by insertion at the pupil location of an Amici Prism $66 \mathrm{~mm}$ long. The prism spreads the $0.45-0.95 \mu \mathrm{m}$ wavelength range on 60 pixels, allowing the recording of 30 2-pixels bins along the range.

\section{The Automatic Quick-Analysis Software}

The Automatic Quick-Analysis software (AQuA) (e.g. Di Paola et al., 2002) has been developed and entirely dedicated to the REM data using criteria of high speed, system stability and reliable results in a fully automated way. It run on a high performance computer with double processor entirely dedicated to REM data handling and analysis. All computers are kindly provided by the italian division of AMD (Advanced Micro Devices, Inc.). AQuA has been conceived to find transient coordinates and colors without any human intervention. Time sensitive data are quickly distributed via internet to the recipients entitled to receive them including $\mathrm{ToO}$ procedure at larger telescopes. When $\mathrm{REM}$ will be operated in the Primary science mode, i.e. following GRB triggers, AQuA will produce 3 classes of output on different time-scales: the coordinate of the IR transient will be available in about 1 minute; magnitudes and colors will be available in a few minutes (possibly giving a rough estimate of the distance of the burst via the photometric red-shift technique); light and color curves will be accurately computed off-line by an extended version of $\mathrm{AQuA}$. Coordinates and colors, expecially in the case of a high-redshift burst, are probably the most valuable science output that REM will provide.

\section{References}

Akerlof C. \& McKay T.A., 1999, IAU Circ., 7100

Costa E. et al., 1997, Nature, 387, 783

Di Paola A., et al. 2002, SPIE, 4847

Fox D. W. 2002, GCN Circ., 1564

van Paradijs J. et al., 1997, Nature, 386, 686

Vitali F. et al., 2002, SPIE, 4841

Zerbi F. M. et al. 2002, SPIE, 4841 